\title{
The Matter Bounce Scenario in Loop Quantum Cosmology
}

\author{
Edward Wilson-Ewing 1,2, * \\ ${ }^{1}$ Aix-Marseille Université, CNRS UMR 7332, CPT, 13288 Marseille, France \\ ${ }^{2}$ Université de Toulon, CNRS UMR 7332, CPT, 83957 La Garde, France
}

\begin{abstract}
In the matter bounce scenario, a dust-dominated contracting space-time generates scale-invariant perturbations that, assuming a nonsingular bouncing cosmology, propagate to the expanding branch and set appropriate initial conditions for the radiation-dominated era. Since this scenario depends on the presence of a bounce, it seems appropriate to consider it in the context of loop quantum cosmology where a bouncing universe naturally arises. For a pressureless collapsing universe in loop quantum cosmology, the predicted power spectrum of the scalar perturbations after the bounce is scale-invariant and the tensor to scalar ratio is negligibly small. A slight red tilt can be given to the scale-invariance of the scalar perturbations by a scalar field whose equation of state is $P=-\epsilon \rho$, where $\epsilon$ is a small positive number. Then, the power spectrum for tensor perturbations is also almost scale-invariant with the same red tilt as the scalar perturbations, and the tensor to scalar ratio is expected to be $r \approx 9 \times 10^{-4}$. Finally, for the predicted amplitude of the scalar perturbations to agree with observations, the critical density in loop quantum cosmology must be of the order $\rho_{c} \sim 10^{-9} \rho_{\mathrm{Pl}}$.
\end{abstract}

PACS numbers: 98.80.Qc, 98.80.Cq

\section{INTRODUCTION}

Observations of the cosmic microwave background (CMB) have shown that the spectrum of scalar perturbations is scale-invariant with a slight red tilt [1] and therefore this is one of the major predictions that any viable cosmological model must make.

There are two well known mechanisms that generate scale invariant perturbations starting from the fluctuations of an initial quantum vacuum state: inflation, an exponential expansion of the universe, and a dustdominated contracting phase. Recent reviews of these two paradigms are 2] and [3], respectively. The fact that they both give rise to a scale-invariant spectra of scalar and tensor perturbations is easily understood as they are related by a simple duality [4].

In this paper, we will focus on the second mechanism. More specifically, we will study the matter bounce scenario where an initially classical contracting dust-dominated universe with quantum vacuum fluctuations gives scale-invariant perturbations once the relevant modes exit the Hubble radius. Assuming the presence of a bounce, the scale-invariant perturbations then provide appropriate initial conditions for the expanding radiation-dominated epoch of our universe.

However, the standard classical treatment of the matter bounce has a major shortcoming in that one must evolve the perturbations from the pre-bounce era to the post-bounce one by hand. Typically this is done by using some reasonable matching conditions [5], but it would be nice to go beyond this and explicitly calculate the propagation of the perturbations through the bounce, where quantum gravity effects are expected to be important.

*Electronic address: wilson-ewing@cpt.univ-mrs.fr
Now, since the matter bounce scenario depends on the presence of a bounce, it is natural to work in the context of loop quantum cosmology (LQC) which predicts a bouncing universe. In this paper, we will study the matter bounce in LQC in order to determine the consequences of quantum gravity effects in this setting. As we shall see, some of the results obtained here are significantly different from those obtained in the matching procedure of [5]; these differences are due to modifications of the Friedmann and Mukhanov-Sasaki equations from quantum gravity effects.

Homogeneous LQC is obtained by following loop quantum gravity (LQG) and using holonomy and flux operators in order to quantize the Hamiltonian constraint corresponding to a homogeneous and isotropic spacetime. See [6, 7] for recent reviews of LQC. One of the main results of LQC is that the classical big-bang singularity is resolved and replaced by a bounce that occurs when the space-time curvature is approximately of the Planck scale. In addition, careful studies of the Friedmann-Lemaître-Robertson-Walker (FLRW) cosmologies in LQC have shown that, for states that are semi-classical (i.e., sharply peaked around a classical solution at late times), there exist a set of effective equations that provide an excellent approximation to the full quantum dynamics of the states at all times, even during the bounce [8, 9].

Cosmological perturbations have also been studied in some detail in LQC, both at the quantum level [10] and especially in the effective theory [11 14]. In this paper we will work with the effective equations for the sake of simplicity. This clearly requires the assumption that the effective equations continue to be a good approximation to the quantum dynamics of semi-classical states in the presence of linear perturbations, which seems reasonable so long as the perturbations remain small.

As an aside, we point out that there are two types of 
corrections, holonomy and inverse triad, that are considered in effective studies in LQC. In this paper we focus on holonomy corrections as they are the dominant ones in the quantum theory of homogeneous LQC and we assume this will continue to be the case when linear perturbations are included. See however [15] for a different perspective.

The paper is organized as follows: in Sec. III we review the effective theory in LQC for both the homogeneous FLRW background and the perturbations. Then we study the dynamics of the cosmological perturbations in a bouncing dust-dominated FLRW universe; scalar perturbations in Sec. III and tensor perturbations in Sec. IV] We close with a discussion in Sec. V]

\section{LOOP QUANTUM COSMOLOGY}

We start by briefly introducing the ingredients coming from loop quantum cosmology that will be necessary for the calculations in the following sections, namely the LQC effective equations. The effective equations are derived from the LQC Hamiltonian constraint operator and include the leading order quantum gravity corrections to the classical Friedmann and Mukhanov-Sasaki equations coming from LQC. It turns out that the effective equations provide a surprisingly good approximation to the dynamics of sharply peaked states in LQC at all times, including at the bounce point where quantum gravity effects are strongest.

Since we are only interested in space-times that are classical (and therefore sharply peaked) at times well before and well after the bounce, we can restrict our attention to semi-classical states in LQC. Because of this we can work in the effective theory, which will considerably simplify the analysis.

An early derivation of the effective equations for the flat FLRW space-time is given in Appendix B of [8], while a considerably more detailed study can be found in $[9]$. Recent reviews of effective equations, including those for some other cosmological space-times, are in Sec. V of [6] and Part III of [7].

We will review the effective equations for the flat FLRW background in the first part of this section, and the effective theory for the scalar and tensor perturbations in the second part.

\section{A. The Homogeneous Background}

Given the flat FLRW metric in terms of the proper time,

$$
\mathrm{d} s^{2}=-\mathrm{d} t^{2}+a(t)^{2} \mathrm{~d} \vec{x}^{2},
$$

where $a(t)$ is the scale factor, and denoting the energy density of the matter field by $\rho$ and its pressure by $P$, the LQC effective equations for the background, including corrections due to quantum geometry effects, are

$$
\begin{aligned}
H^{2} & =\frac{8 \pi G}{3} \rho\left(1-\frac{\rho}{\rho_{c}}\right), \\
\frac{\ddot{a}}{a}-H^{2} & =-4 \pi G(\rho+P)\left(1-\frac{2 \rho}{\rho_{c}}\right), \\
\dot{\rho} & =-3 H(\rho+P),
\end{aligned}
$$

where $\rho_{c}$ is the critical energy density and $H=\dot{a} / a$ is the Hubble rate. The dot denotes differentiation with respect to the proper time $t$. Finally, note that the classical Friedmann equations are recovered in the limit of $\rho_{c} \rightarrow$ $\infty$.

When the matter field is a perfect fluid with a constant equation of state $P=\omega \rho$, these equations can be solved, giving

$$
\begin{aligned}
\rho & =\rho_{o} a^{-3(1+\omega)}, \\
a(t) & =\left(6 \pi G \rho_{o}(1+\omega)^{2}\left(t-t_{o}\right)^{2}+\frac{\rho_{o}}{\rho_{c}}\right)^{\frac{1}{3(1+\omega)}} .
\end{aligned}
$$

From the nonvanishing form of $a(t)$, it is clear that the big bang singularity is resolved and replaced by a bounce. The dimensionful constants of integration $\rho_{o}$ and $t_{o}$, which determine the magnitude of the scale factor and the time the bounce occurs at, will be set to $\rho_{o}=\rho_{c}$ and $t_{o}=0$ so that $a\left(t_{\text {bounce }}\right)=1$ and $t_{\text {bounce }}=0$; these choices do not affect the physics.

As an aside, it is worth pointing out that while the scale factor $a$ can be expressed in a relatively simple manner in terms of the proper time $t$, this is not true when one works in conformal time $\eta$, defined by

$$
\mathrm{d} \eta=\frac{\mathrm{d} t}{a(t)}
$$

in which case the form of the scale factor is considerably more complicated. For this reason, we will try to work in proper time whenever LQC effects are important. On the other hand, it will be useful to work in conformal time in order to study the perturbations in the classical limit and therefore we will switch between the two different time choices depending on the situation.

Finally, if one is working with a scalar field, then the energy density and the pressure are given by the same relations as in the classical theory,

$$
\rho=\frac{1}{2} \dot{\varphi}^{2}+V(\varphi), \quad P=\frac{1}{2} \dot{\varphi}^{2}-V(\varphi) .
$$

It is possible, for the potential ${ }^{1}$

$$
V(\varphi)=\frac{(1-\omega) V_{o} e^{-\sqrt{24 \pi G(1+\omega)} \varphi}}{\left(1+\frac{V_{o}}{2 \rho_{c}} e^{-\sqrt{24 \pi G(1+\omega)} \varphi}\right)^{2}}
$$

\footnotetext{
${ }^{1}$ Note that the denominator in the potential is necessary due to the modifications in the LQC Friedmann equations. The usual potential used to get a constant equation of state given in e.g. [5] is obtained in the limit $\rho_{c} \rightarrow \infty$.
} 
to mimic a cosmology with a constant equation of state $P=\omega \rho$ by using a scalar field, so long as one starts with appropriate initial conditions [16]. This is what will be done here, for the case of $\omega=0$.

\section{B. Perturbations}

Classically, scalar perturbations in cosmology can be studied by using the Mukhanov-Sasaki equation. (For an introduction to the theory of cosmological perturbations in the classical theory, see for example [17, 18].) In LQC, there are modifications to the equations of motion governing the dynamics of the perturbations, just as the Friedmann equations are modified. The corrections have been studied for the cases of a perfect fluid [11] and a scalar field [12, 13]. Here we are working with a scalar field, in which case the modified Mukhanov-Sasaki equation is

$$
v^{\prime \prime}-\left(1-\frac{2 \rho}{\rho_{c}}\right) \nabla^{2} v-\frac{z^{\prime \prime}}{z} v=0,
$$

where the prime denotes differentiation with respect to the conformal time $\eta$,

$$
v=a \sqrt{\rho+P} \delta u^{(g i)}-z \Phi,
$$

where $\delta u^{(g i)}$ and $\Phi$ are the usual gauge-invariant observables in cosmological perturbation theory and

$$
z=\frac{a}{H} \sqrt{(\rho+P)} .
$$

Note that although (12) has the same form as in classical general relativity, the dynamics of all of the background variables are modified in LQC and $z$ will behave very differently near the bounce as compared to what might be expected classically. In particular, if the background dynamics are those of a perfect fluid with a constant equation of state $P=\omega \rho$ as is the case for the matter bounce scenario, then Eq. (2) shows that there is an extra term coming from LQC in the denominator,

$$
z=\sqrt{\frac{3(1+\omega)}{8 \pi G}} \cdot \frac{a}{\sqrt{1-\rho / \rho_{c}}} .
$$

The Mukhanov-Sasaki equation is most easily solved in Fourier space, where it becomes

$$
v_{k}^{\prime \prime}+\left(1-\frac{2 \rho}{\rho_{c}}\right) k^{2} v_{k}-\frac{z^{\prime \prime}}{z} v_{k}=0 .
$$

Tensor perturbations behave a little differently: their dynamics are governed by an equation that has the same form as for scalar perturbations, but even classically the variables are defined slightly differently, and it turns out that the quantum geometry corrections do not appear in exactly the same manner either. The holonomy-corrected
Mukhanov-Sasaki equation for tensor perturbations in Fourier space is given by [14]

$$
\mu_{k}^{\prime \prime}+\left(1-\frac{2 \rho}{\rho_{c}}\right) k^{2} \mu_{k}-\frac{z_{T}^{\prime \prime}}{z_{T}} \mu_{k}=0,
$$

where

$$
z_{T}=\frac{a}{\sqrt{1-2 \rho / \rho_{c}}},
$$

and $\mu_{k}=z_{T} h_{k}$, with $h$ being the usual gravitational wave perturbation variable. Note that there is no need for absolute values in the denominator of $z_{T}$ as $\mu_{k}$ is a complex variable. The fact that there are no absolute values will be important in Sec. IV.

Finally, one might be worried about the divergences in $z$ and $z_{T}$ that occur at or near the bounce point. However, we shall see that the solutions for $v_{k}$ and $\mu_{k}$ can be determined despite these divergences and, assuming small perturbations before the bounce, the perturbations are small after the bounce as well. Therefore, these divergences do not prevent us from obtaining explicit solutions for $v_{k}$ and $\mu_{k}$ and studying their properties after the bounce.

A separate open question is whether the divergences in $z$ and $z_{T}$ can drive the perturbations to become large for a short period of time close to the bounce, at which point back reaction effects might become important. We leave a more careful study of this issue for future work.

For ease of notation, we will drop the index $k$ in (14) and (15) in the future.

\section{SCALAR PERTURBATIONS}

We will begin by studying the propagation of scalar perturbations in an LQC dust-dominated universe. As perturbations are most easily studied in conformal time, and the initial conditions are to be set in a classical regime where quantum gravity effects are negligible, it is useful to recall the classical relations giving the scale factor and the proper time in terms of the conformal time for $P=0$,

$$
a(\eta)=\frac{2 \pi G \rho_{c}}{3} \eta^{2}, \quad t(\eta)=\frac{2 \pi G \rho_{c}}{9} \eta^{3} .
$$

Another useful relation is $z(\eta)$ in the classical limit, given by

$$
z(\eta)=\sqrt{\frac{\pi G}{6}} \rho_{c} \eta^{2} .
$$

We will now study how the perturbations propagate through the bounce by imposing quantum vacuum initial conditions in the pre-bounce phase and then determining the form of the perturbations in the post-bounce phase. This will be done by matching the classical solutions, in both the pre- and post-bounce eras, order by order with a formal solution given by an expansion in $k$ that holds at all times, including at the bounce point. 


\section{A. The Contracting Branch and the Bounce}

In the classical limit, and for a dust-dominated universe, (14) simplifies to

$$
v^{\prime \prime}+\left(k^{2}-\frac{2}{\eta^{2}}\right) v=0 .
$$

If (19) is rewritten for $f=v / \sqrt{-\eta}$ and the time variable is rescaled by a factor of $k$, this becomes the Bessel differential equation for $f$, and thus the solution to (19) is given by

$$
v(\eta)=\sqrt{-\eta}\left[A_{1} H_{3 / 2}^{(1)}(-k \eta)+A_{2} H_{3 / 2}^{(2)}(-k \eta)\right],
$$

where $H_{n}^{(1)}(x)$ and $H_{n}^{(2)}(x)$ are the Hankel functions, while $A_{1}$ and $A_{2}$ are constants to be determined by the initial conditions. Note the presence of a minus sign in front of $\eta$ as this solution holds in the contracting branch, where the time variables are negative.

Now, by the asymptotic behaviour of the Hankel functions, we find that (up to an irrelevant global phase which we leave out)

$$
\lim _{\eta \rightarrow-\infty} v(\eta) \sim A_{1} \sqrt{\frac{2}{\pi k}} e^{-i k \eta}+A_{2} \sqrt{\frac{2}{\pi k}} e^{i k \eta},
$$

and therefore it is possible to impose the vacuum initial conditions

$$
v_{\text {initial }}=\sqrt{\frac{\hbar}{2 k}} e^{-i k \eta},
$$

by choosing $A_{1}=\sqrt{\pi \hbar / 4}$ and $A_{2}=0$, which gives

$$
v(\eta)=\sqrt{\frac{-\pi \hbar \eta}{4}} H_{3 / 2}^{(1)}(-k \eta) .
$$

This gives the expression of the Mukhanov variable so long as quantum geometry effects are negligible.

Of course, as the space-time contracts, the curvature will increase and at some point LQC effects will begin to play an important role. Therefore it will be necessary to match this solution to another one which will be valid throughout the bounce.

This can be done by rewriting (14) in an integral form, i.e., as

$$
\begin{aligned}
v(\eta)= & B_{1} z+B_{2} z \int^{\eta} \frac{\mathrm{d} \bar{\eta}}{z^{2}}-k^{2} z \int^{\eta} \frac{\mathrm{d} \bar{\eta}}{z^{2}} \int^{\bar{\eta}} \mathrm{d} \overline{\bar{\eta}} z v \\
& +\frac{2 k^{2}}{\rho_{c}} z \int^{\eta} \frac{\mathrm{d} \bar{\eta}}{z^{2}} \int^{\bar{\eta}} \mathrm{d} \overline{\bar{\eta}} \rho z v .
\end{aligned}
$$

Doing an expansion in $k$, we immediately find that the leading order terms are

$$
v(\eta)=B_{1}\left(z+O\left(k^{2}\right)\right)+B_{2}\left(z \int^{\eta} \frac{\mathrm{d} \bar{\eta}}{z^{2}}+O\left(k^{2}\right)\right) .
$$

An important point here is that the constants $B_{1}$ and $B_{2}$ can (and will) depend on $k$. For a dust-dominated space-time in LQC,

$$
a(t)=\left(6 \pi G \rho_{c} t^{2}+1\right)^{1 / 3}, \quad z(t)=\frac{a(t)^{5 / 2}}{4 \pi G \sqrt{\rho_{c}} t},
$$

and by using (7) we find that the two leading order terms in $k$ of $v(t)$ are given by

$$
\begin{gathered}
v(t)=B_{1} z(t)+\left[\sqrt{\frac{8 \pi G}{27 \rho_{c}}}\left(\arctan \sqrt{6 \pi G \rho_{c}} t+\frac{\pi}{2}\right)\right. \\
\left.-\frac{4 \pi G t}{3\left(6 \pi G \rho_{c} t^{2}+1\right)}\right] B_{2} z(t),
\end{gathered}
$$

where the constant of integration has been chosen in order to simplify the matching of this solution with (23). Indeed, in the classical regime of the contracting branch where $t \ll-1 / \sqrt{6 \pi G \rho_{c}}$, we find that

$$
v(t)=B_{1} z(t)-\frac{4 B_{2} z(t)}{9 \rho_{c} t},
$$

and it is possible to match this solution with (23) by ensuring that the coefficients of the leading terms in $k$ match in the classical regime.

We cannot take the limit $\eta \rightarrow 0$ of (23) as quantum gravity effects are important in this regime and then the classical solution cannot be trusted. Instead, we will consider the limit of small $k \eta$ at a time where general relativity can be trusted. Note that this limit can only be taken for the modes that have become larger than the Hubble radius before LQC effects become important. However, in the matter bounce scenario all of the modes that we can observe in the CMB today satisfy this property and therefore this limitation is not problematic. In the small $k \eta$ limit, $v(\eta)$ contributes two terms, each of which can be used to fix one of $B_{1}$ and $B_{2}$.

In the $k \eta \ll 1$ limit, the solution (23) tends to

$$
v(\eta) \rightarrow \frac{\sqrt{\hbar}}{3 \sqrt{2}} k^{3 / 2} \eta^{2}-\frac{i \sqrt{\hbar}}{\sqrt{2} k^{3 / 2} \eta},
$$

and therefore the constants $B_{1}$ and $B_{2}$ must be taken to be

$$
B_{1}=\frac{\sqrt{\hbar}}{\sqrt{3 \pi G} \rho_{c}} k^{3 / 2}, \quad B_{2}=i \frac{\sqrt{3 \pi G \hbar}}{2} \rho_{c} k^{-3 / 2} .
$$

\section{B. The Expanding Branch}

We now know the form of $v(t)$, at least in the form of the formal solution (24), and from this it is possible to determine the spectrum of scalar perturbations after the bounce.

In order to do this, we will again work with the leading order terms of the formal solution to $v$, given by (27). For 
times after the bounce where quantum gravity effects are negligible, $t \gg 1 / \sqrt{6 \pi G \rho_{c}}$ and then the behaviour of $v(t)$ is, for the leading order terms in $k$,

$$
v(t)=\left(B_{1}+\sqrt{\frac{8 \pi^{3} G}{27 \rho_{c}}} B_{2}\right) z(t)-\frac{4 B_{2} z(t)}{9 \rho_{c} t},
$$

and we see that a mixing has occurred in the first term. It is this new term, coming from the mixing, that will give a scale-invariant spectrum. This is what we shall show now.

In order to have the full solution to $v(\eta)$, the easiest method is to use the classical Mukhanov-Sasaki equation (19) that holds once LQC effects become small after the bounce. The classical solution, to all orders in $k$, is given by

$$
v(\eta)=\sqrt{\eta}\left[C_{1} H_{3 / 2}^{(1)}(k \eta)+C_{2} H_{3 / 2}^{(2)}(k \eta)\right],
$$

where $C_{1}$ and $C_{2}$ are two constants that must be determined by matching the lowest order terms in $k$ of this solution with (31).

This matching gives

$$
v(\eta)=\sqrt{\pi \hbar \eta}\left[\frac{1}{2} H_{3 / 2}^{(2)}(k \eta)+i \frac{\pi^{2}\left(G \rho_{c}\right)^{3 / 2}}{\sqrt{6} k^{3}} J_{3 / 2}(k \eta)\right],
$$

where $J_{n}(x)$ is the Bessel function of the first kind and it is understood that the prefactors have been determined to leading order in $k$.

Therefore, at times after the bounce when quantum gravity effects are negligible (but before the modes reenter the Hubble radius), the perturbations look like

$$
\begin{aligned}
v(\eta)= & \frac{i \sqrt{\hbar}}{k^{3 / 2}}\left[\pi^{5 / 2}\left(\frac{G \rho_{c}}{3}\right)^{3 / 2} \eta^{2}-\frac{1}{\sqrt{2} \pi \eta}\right] \\
& +\frac{\sqrt{\hbar}}{3 \sqrt{2} \pi} k^{3 / 2} \eta^{2} .
\end{aligned}
$$

In order to calculate the amplitude of the scalar fluctuations, we can use the variable $\mathcal{R}$ which measures the curvature perturbations and is related to $v$ simply by [17, 18],

$$
\mathcal{R}(\eta)=\frac{v}{z} \sim i \frac{\sqrt{2 \pi^{4} G^{2} \hbar \rho_{c}}}{3} k^{-3 / 2},
$$

where we have only kept the dominant mode in terms of $k$, and also dropped the mode that decays as $\eta$ grows. From this, it is possible to calculate the scalar power spectrum given by

$$
\Delta_{\mathcal{R}}^{2}(k)=\frac{k^{3}}{2 \pi^{2}}|\mathcal{R}(\eta)|^{2} \sim \frac{\pi^{2} G^{2} \hbar \rho_{c}}{9} k^{0} .
$$

This shows that the scalar spectral index is $n_{s}=1$, and thus the power spectrum is scale-invariant.

For the amplitude of the scalar perturbations given in (36) to agree with the observed value of $\Delta_{\mathcal{R}}^{2} \sim 10^{-9}$
[1], it is necessary for the critical density to be of the order of $10^{-9} \rho_{\mathrm{Pl}}$. This seems to contradict LQC where the critical energy density is usually assumed to be of the order of the Planck density, in which case the matter bounce scenario would be ruled out in LQC. However, it is important to remember that the numerical value of the critical energy density must ultimately be derived from loop quantum gravity, and this remains an open problem. The critical energy density may turn out to be much smaller than expected, in which case the matter bounce scenario would be viable in LQC.

\section{The Equation of State $P=-\epsilon \rho$}

A slight red tilt to the spectrum of scalar perturbations is obtained by working with the equation of state $P=-\epsilon \rho$, where $0<\epsilon \ll 1$. We will not go through all of the details of the calculation here as they are a straightforward extension of what is done earlier in this section.

It is a relatively simple task to determine the spectrum of scalar perturbations for this new equation of state (the calculations simplify considerably if terms of order $\epsilon^{2}$ and higher are dropped). The resulting spectrum has a scalar index of

$$
n_{s}=1-12 \epsilon,
$$

which given the observed scalar index of $n_{s}=0.968 \pm$ 0.012 [1], implies that we must have

$$
\epsilon \approx 0.003
$$

which validates the approximation $\epsilon \ll 1$.

Thus, it is possible to match the observed red tilt of the scalar perturbation spectrum by choosing an appropriate matter field. In the next section, where we shall study tensor perturbations, we will exclusively work with a background where the equation of state is $P=-\epsilon \rho$, as this is the relevant setting whose predictions agree with observations.

\section{TENSOR PERTURBATIONS}

For tensor perturbations, we will follow the same procedure as in the previous section, i.e., impose quantum vacuum initial conditions before the bounce and use matching conditions in order to determine the form of the tensor perturbations after the bounce.

One difference with the previous section is that we will work in the setting where the equation of state is $P=$ $-\epsilon \rho$, with $0<\epsilon \ll 1$. This is the background that gives a slight red tilt to the spectrum of scalar perturbations. In addition, as we shall see, for tensor perturbations the limit of $\epsilon=0$ gives a qualitatively different result so it is important to work from the start with a nonzero value 
for $\epsilon$. Because $\epsilon$ is very small, we will neglect all terms that are of the order of $\epsilon^{2}$ or smaller.

To first order in $\epsilon$, the scale factor and the proper time in the classical theory, in terms of the conformal time, are given by

$$
\begin{gathered}
a(\eta)=\left((1-6 \epsilon) \frac{2 \pi G \rho_{c}}{3} \eta^{2}\right)^{1+3 \epsilon}, \\
t(\eta)=\left(6 \pi G \rho_{c}\right)^{1+3 \epsilon}\left(\frac{1-2 \epsilon}{3} \eta\right)^{3(1+2 \epsilon)} .
\end{gathered}
$$

While it is possible to expand the exponents around $\epsilon=0$, this is not necessary and would merely complicate calculations later.

\section{A. The Contracting Branch and the Bounce}

In the classical limit, the Mukhanov-Sasaki equation for tensor perturbations with an equation of state $P=$ $-\epsilon \rho$ is given by

$$
\mu^{\prime \prime}+\left(k^{2}-\frac{2(1+9 \epsilon)}{\eta^{2}}\right) \mu=0,
$$

and choosing the initial conditions to be the quantum vacuum state (22) gives

$$
\mu(\eta)=\sqrt{\frac{-\pi \hbar \eta}{4}} H_{n}^{(1)}(-k \eta)
$$

in the classical regime of the contracting branch of the cosmology with

$$
n=\frac{3}{2}+6 \epsilon .
$$

As before, we will link the solutions in the contracting and expanding branches via the leading order solution in $k$ by using the integral form of the modified MukhanovSasaki equation,

$$
\begin{aligned}
\mu(\eta)= & D_{1} z_{T}+D_{2} z_{T} \int^{\eta} \frac{\mathrm{d} \bar{\eta}}{z_{T}^{2}}-k^{2} z_{T} \int^{\eta} \frac{\mathrm{d} \bar{\eta}}{z_{T}^{2}} \int^{\bar{\eta}} \mathrm{d} \overline{\bar{\eta}} z_{T} \mu \\
& +\frac{2 k^{2}}{\rho_{c}} z_{T} \int^{\eta} \frac{\mathrm{d} \bar{\eta}}{z_{T}^{2}} \int^{\bar{\eta}} \mathrm{d} \overline{\bar{\eta}} \rho z_{T} \mu
\end{aligned}
$$

and solving for the two leading order terms in $k$.

For $\omega=-\epsilon$ in $\mathrm{LQC}$,

$$
z_{T}(t)=\frac{\left[6 \pi G \rho_{c}(1-2 \epsilon) t^{2}+1\right]^{\frac{5}{2}+\frac{3 \epsilon}{2}}}{\sqrt{6 \pi G \rho_{c}(1-2 \epsilon) t^{2}-1}},
$$

and therefore we find that the two leading order terms in $k$ of $\mu$ are

$$
\begin{gathered}
\mu(t)=D_{1} z_{T}(t)-\left[\frac{\epsilon}{\sqrt{6 \pi G \rho_{c}}}\left(\arctan \sqrt{6 \pi G \rho_{c}} t+\frac{\pi}{2}\right)\right. \\
\left.+\frac{(1-\epsilon) t}{\left[6 \pi G \rho_{c}(1-2 \epsilon) t^{2}+1\right]^{1+\epsilon}}\right] D_{2} z_{T}(t)
\end{gathered}
$$

where the integration constant is again chosen in order to simplify the matching procedure. Then, in the regime $t \ll-1 / \sqrt{6 \pi G \rho_{c}}$, the expression for $\mu(t)$ simplifies to

$$
\mu(t)=D_{1} a(t)-\frac{(1-2 \epsilon) D_{2} a(t)}{\left[6 \pi G \rho_{c}(1-2 \epsilon)\right]^{1+\epsilon} t^{1+2 \epsilon}},
$$

and therefore, for the two solutions to match, we must have

$$
\begin{aligned}
& D_{1}=\sqrt{\frac{8 \hbar}{9}}\left(\frac{3}{8 \pi G \rho_{c}}\right)^{1+3 \epsilon} k^{n}, \\
& D_{2}=i \sqrt{\frac{9 \hbar}{32}}\left(\frac{8 \pi G \rho_{c}}{3}\right)^{1+3 \epsilon} k^{-n},
\end{aligned}
$$

where we have dropped the nonexponential dependence of $\epsilon$ in the prefactors as this will not be relevant for our calculations.

\section{B. The Expanding Branch}

In order to determine the spectrum of gravitational waves in the classical regime of the expanding branch after the bounce, it is necessary to match the classical solution

$$
\mu(\eta)=\sqrt{\eta}\left[E_{1} H_{n}^{(1)}(k \eta)+E_{2} H_{n}^{(2)}(k \eta)\right]
$$

to leading order in $k$ with the leading order terms of the formal solution (46) in the regime $t \gg 1 / \sqrt{6 \pi G \rho_{c}}$, where the two leading order terms have the form

$$
\begin{aligned}
\mu(t)= & \left(D_{1}-\sqrt{\frac{\pi}{6 G \rho_{c}}} \epsilon D_{2}\right) a(t) \\
& -\frac{(1-2 \epsilon) D_{2} a(t)}{\left[6 \pi G \rho_{c}(1-2 \epsilon)\right]^{1+\epsilon} t^{1+2 \epsilon}} .
\end{aligned}
$$

From this expression, and the previous calculations, one immediately sees that

$$
\begin{aligned}
\mu(\eta)= & \sqrt{\pi \hbar \eta}\left[-i \frac{\epsilon}{32 k^{2 n}} \sqrt{\frac{27 \pi}{2 G \rho_{c}}}\left(\frac{8 \pi G \rho_{c}}{3}\right)^{2+6 \epsilon} J_{n}(k \eta)\right. \\
& \left.+\frac{1}{2} H_{n}^{(2)}(k \eta)\right]
\end{aligned}
$$

where the prefactors are understood to be accurate to leading order in $k$. Again, just as in the case of scalar perturbations, a new mode is created by the bounce. As the amplitude of the tensor perturbations is of the order of $\epsilon$, this will give a small tensor to scalar ratio.

From the expression of $\mu$, it is possible to determine $h$,

$$
h=\frac{\mu}{a} \sim-i \frac{\epsilon \pi}{2} \sqrt{\frac{\hbar}{2}}\left(\frac{8 \pi G \rho_{c}}{3}\right)^{\frac{1}{2}+3 \epsilon} k^{-\frac{3}{2}-6 \epsilon},
$$

where the first equality holds in the classical regime and we have kept only the constant part of the leading order term in $k$ on the righthand side. 

by $^{2}$

The amplitude of the tensor perturbations is then given

$$
\begin{aligned}
\Delta_{h}^{2}(k) & =64 \pi G \frac{k^{3}}{2 \pi^{2}}|h|^{2} \\
& =\epsilon^{2} \frac{32 \pi^{2} G^{2} \hbar \rho_{c}}{3}\left(\frac{8 \pi G \rho_{c}}{3 k^{2}}\right)^{6 \epsilon}
\end{aligned}
$$

and thus we get a spectrum of tensor perturbations with a small amplitude and an almost scale-invariant spectrum with $n_{T}=-12 \epsilon$.

Finally, it is possible to determine the tensor to scalar ratio, given by

$$
r=\frac{\Delta_{h}^{2}}{\Delta_{\mathcal{R}}^{2}}=96 \epsilon^{2} \approx 9 \times 10^{-4},
$$

where we have inserted the value of $\epsilon$ in (38), which was determined by the observed tilt of the spectrum of scalar perturbations. The predicted value for $r$ is so small that, if the matter bounce scenario is the correct one, we do not expect to observe a primordial gravitational wave background until the precision of astronomical observations increases by an order of magnitude or two.

It is worth noting that it is important to work with the equation of state $P=-\epsilon \rho$ for tensor perturbations as the predictions are significantly different than for a pure dust matter field with $P=0$ : for the case $P=0$, no new mode appears in (52) and then the resulting spectrum would have a strong blue tilt of $n_{T}=6$ (and therefore primordial gravitational waves would not be observable as their amplitude would be proportional to $\ell_{\mathrm{Pl}}^{6} k^{6}$, an extremely small factor for all relevant modes). Thus, the small deviation from a dust fluid plays an important role as it generates the new mode in (52) which is scaleinvariant and the only one which is potentially observable today in this scenario.

\section{DISCUSSION}

In loop quantum cosmology, quantum gravity effects modify both the Friedmann equations and the Mukhanov-Sasaki equations, which govern the dynamics of the homogeneous background and of the linear perturbations respectively. These modifications become important at large curvature scales, and especially at the bounce point. Therefore, it is important to include these effects in any setting where the cosmological bounce plays a role, and this is what has been done here for the matter bounce scenario.

The matter bounce in loop quantum cosmology gives a scale-invariant spectrum for scalar and tensor perturbations, and a slight red tilt is obtained for the equation

\footnotetext{
2 There is an extra factor of $32 \pi G$ in the definition of $\Delta_{h}^{2}$ for dimensional reasons, and another additional factor of 2 to account for the two polarizations. See e.g. [2] for details.
}

of state $P=-\epsilon \rho$, with $0<\epsilon \ll 1$. The same tilt is predicted for the scalar and tensor modes. As an identical tilt for the scalar and tensor modes is not expected in inflationary models, this is one way the two scenarios can be differentiated.

The other main results are that the observed amplitude for scalar perturbations is obtained for a value of the critical energy density of $\rho_{c} \sim 10^{-9} \rho_{\mathrm{Pl}}$, and a tensor to scalar ratio is predicted to be $r \approx 9 \times 10^{-4}$. Note that there are some important differences between these predictions for the matter bounce scenario and those given in [5] where quantum gravity effects were not included, particularly regarding the amplitudes of the spectra and their relative importance.

Although the value of $\rho_{c}$ is usually assumed to be within one order of magnitude of the Planck energy density, this is a quantity that should be derived from loop quantum gravity. Thus, until the relation between the full theory of LQG and its cosmological sector is better understood, the critical energy density remains an unknown, and may turn out to be considerably smaller than expected. In any case, it is clear that for the matter bounce scenario to be viable in LQC we must have $\rho_{c} \sim 10^{-9} \rho_{\mathrm{Pl}}$.

One of the key predictions here is the small tensor to scalar ratio, which is proportional to $\epsilon^{2}$ (and is therefore related to $n_{s}$ ). The modifications to the scalar and tensor Mukhanov-Sasaki equations are slightly different, and this plays a major role in the matter bounce scenario as one of its effects is the much smaller amplitude of the tensor perturbations than that predicted from classical considerations. The reason why the amplitude of the tensor perturbations is so small (and vanishes in the limit $\epsilon \rightarrow 0$ ) is that $z_{T}$, defined in (16), becomes imaginary near the bounce. Therefore, the integral $\int \mathrm{d} \eta / z_{T}^{2}$ can vanish as some portions of it will be negative. On the other hand, the integrand in $\int \mathrm{d} \eta / z^{2}$ [see (13)] is always positive so the integral cannot vanish. This is why the tensor to scalar ratio is suppressed for the matter bounce scenario in LQC. The difference between the LQC modifications to the scalar and tensor Mukhanov-Sasaki equations is one way that quantum gravity effects can significantly change results obtained in a purely classical setting.

It is interesting to compare these results to those obtained for the matter bounce scenario in different cosmological models. Two matter bounce cosmologies that give similar predictions are (i) with a Lee-Wick type scalar field that causes a bounce [19] (the case where the LeeWick scalar is non-minimally coupled to gravity is studied in [20]), and (ii) in the setting of the $f(T)$ generalization of teleparallel gravity [21]. It is possible to incorporate the matter bounce scenario in both of these cases, and then the spectra for the scalar and tensor perturbations after the bounce will be scale-invariant. However, the main shortcoming of both of these models is that they predict a tensor to scalar ratio that is approximately unity (at least for the typical implementation of these models), thus violating the bound of $r \lesssim 0.25$ given 
in [1]. Another setting the matter bounce scenario has been considered in is that of Bohmian quantum cosmology [22]. Although a small tensor to scalar ratio is obtained in this case, the spectra for the scalar and tensor perturbations are expected to have a slight blue (rather than red) tilt. It would be possible to obtain a slight red tilt by using a scalar field rather than dust as the matter content in the Bohmian quantum cosmology, but then the resulting scalar to tensor ratio would be close to unity. In the matter bounce scenario, it is often difficult to obtain the two characteristics of a red tilt in the spectrum of scalar perturbations and a small tensor to scalar ratio. As we have seen, this is not the case in LQC.

A more promising matter bounce alternative is studied in [23], where a combination of ingredients coming from the matter bounce and ekpyrotic cosmological scenarios is used in order to obtain scale-free perturbations and also a small scalar to tensor ratio. In this setting, the small value of $r$ arises because the sound speed of the scalar perturbations becomes negative for a short time and this generates exponential growth in the amplitude of the scalar perturbations. Since the speed sound of the tensor perturbations remains positive, their magnitude is unchanged and thus $r$ is small. Note that this is the opposite mechanism of what occurs in LQC: in LQC, it is the negative value of $z_{T}^{2}$ during the bounce which damps the amplitude of the tensor modes, while in this other scenario it is the negative speed sound that generates growth in the scalar modes. An important point is that this cosmology can be differentiated from the one studied in this paper by observations as the predicted scalar to tensor ratio in 23] is $10^{-5}$ (although the predicted value of $r$ in this setting is model dependent to some extent), almost two orders of magnitude smaller than what is predicted in LQC. This is the main difference between the predictions of the two cosmologies.

Finally, it is reasonable to expect that quantum gravity effects could also give nontrivial corrections to the classical predictions of other cosmological models, including inflation. As it is known how to include inflation in LQC [24, 25], it is important now to study how quantum gravity effects could affect the standard inflationary predictions. Some work has already been done in this direction, see for example [15, 26].

As the effects of LQC and other quantum cosmology models on the $\mathrm{CMB}$ and primordial gravitational waves are better understood, and the observations continue to improve, it will become possible to differentiate between (i) alternative cosmological scenarios (inflation, matter bounce, etc.), and (ii) the various quantum cosmology theories (LQC, string cosmology, etc.), and thus determine which combination of the two is realized in nature.

\section{Acknowledgments}

The author would like to thank Ivan Agulló, Thomas Cailleteau and William Nelson for helpful discussions. This work was supported by Le Fonds québécois de la recherche sur la nature et les technologies.
[1] WMAP Collaboration, E. Komatsu et al., "Seven-Year Wilkinson Microwave Anisotropy Probe (WMAP) Observations: Cosmological Interpretation," Astrophys. J. Suppl. 192 (2011) 18, arXiv:1001.4538.

[2] D. Baumann, "TASI Lectures on Inflation," arXiv:0907.5424

[3] R. H. Brandenberger, "The Matter Bounce Alternative to Inflationary Cosmology," arXiv:1206.4196.

[4] D. Wands, "Duality invariance of cosmological perturbation spectra," Phys. Rev. D60 (1999) 023507, arXiv: gr-qc/9809062

[5] F. Finelli and R. Brandenberger, "On the generation of a scale invariant spectrum of adiabatic fluctuations in cosmological models with a contracting phase," Phys. Rev. D65 (2002) 103522, arXiv: hep-th/0112249

[6] A. Ashtekar and P. Singh, "Loop Quantum Cosmology: A Status Report," Class. Quant. Grav. 28 (2011) 213001, arXiv:1108.0893

[7] K. Banerjee, G. Calcagni, and M. Martín-Benito, "Introduction to loop quantum cosmology," SIGMA 8 (2012) 016, arXiv:1109.6801

[8] A. Ashtekar, T. Pawłowski, and P. Singh, "Quantum Nature of the Big Bang: Improved dynamics," Phys. Rev. D74 (2006) 084003, arXiv:gr-qc/0607039

[9] V. Taveras, "Corrections to the Friedmann Equations from LQG for a Universe with a Free Scalar Field," Phys. Rev.
D78 (2008) 064072, arXiv:0807.3325

[10] E. Wilson-Ewing, "Lattice loop quantum cosmology: scalar perturbations," Class. Quant. Grav. 29 (2012) 215013, arXiv:1205.3370.

[11] E. Wilson-Ewing, "Holonomy Corrections in the Effective Equations for Scalar Mode Perturbations in Loop Quantum Cosmology," Class. Quant. Grav. 29 (2012) 085005, arXiv:1108.6265.

[12] T. Cailleteau, J. Mielczarek, A. Barrau, and J. Grain, "Anomaly-free scalar perturbations with holonomy corrections in loop quantum cosmology," Class. Quant. Grav. 29 (2012) 095010, arXiv:1111.3535.

[13] T. Cailleteau and A. Barrau, "Gauge invariance in Loop Quantum Cosmology : Hamilton-Jacobi and Mukhanov-Sasaki equations for scalar perturbations," Phys. Rev. D85 (2012) 123534, arXiv:1111.7192,

[14] T. Cailleteau, A. Barrau, J. Grain, and F. Vidotto, "Consistency of holonomy-corrected scalar, vector and tensor perturbations in Loop Quantum Cosmology," Phys. Rev. D86 (2012) 087301, arXiv:1206.6736.

[15] M. Bojowald, G. Calcagni, and S. Tsujikawa, "Observational test of inflation in loop quantum cosmology," JCAP 1111 (2011) 046, arXiv:1107.1540.

[16] J. Mielczarek, "Multi-fluid potential in the loop cosmology," Phys. Lett. B675 (2009) 273-278, arXiv:0809.2469

[17] V. F. Mukhanov, H. Feldman, and R. H. Brandenberger, 
"Theory of cosmological perturbations," Phys. Rept. 215 (1992) 203-333.

[18] P. Peter and J.-P. Uzan, Primordial Cosmology. Oxford University Press, 2009.

[19] Y.-F. Cai, T.-T. Qiu, R. Brandenberger, and X.-M. Zhang, "A Nonsingular Cosmology with a Scale-Invariant Spectrum of Cosmological Perturbations from Lee-Wick Theory," Phys. Rev. D80 (2009) 023511, arXiv:0810.4677

[20] T. Qiu and K.-C. Yang, "Perturbations in Matter Bounce with Non-minimal Coupling," JCAP 1011 (2010) 012, arXiv:1007.2571

[21] Y.-F. Cai, S.-H. Chen, J. B. Dent, S. Dutta, and E. N. Saridakis, "Matter Bounce Cosmology with the $\mathrm{f}(\mathrm{T})$ Gravity," Class. Quant. Grav. 28 (2011) 215011, arXiv:1104.4349.

[22] P. Peter, E. J. Pinho, and N. Pinto-Neto, "A Non inflationary model with scale invariant cosmological perturbations," Phys. Rev. D75 (2007) 023516, arXiv:hep-th/0610205

[23] Y.-F. Cai, D. A. Easson, and R. Brandenberger, "Towards a Nonsingular Bouncing Cosmology," JCAP 1208 (2012) 020, arXiv: 1206.2382

[24] A. Ashtekar and D. Sloan, "Probability of Inflation in Loop Quantum Cosmology," Gen. Rel. Grav. 43 (2011) 3619-3655, arXiv:1103.2475.

[25] M. Fernandez-Mendez, G. A. Mena Marugan, and J. Olmedo, "Hybrid quantization of an inflationary universe," Phys. Rev. D86 (2012) 024003, arXiv:1205.1917

[26] I. Agullo, A. Ashtekar, and W. Nelson, "A Quantum Gravity Extension of the Inflationary Scenario," arXiv:1209.1609. 